\title{
ARTICLE
}

\section{High-dose antipsychotics: addressing patients' resistance to physical health monitoring}

\author{
Paula Marie Murphy, Andrew Iles \& Suja Sreedharan
}

Paula Murphy and Andrew lles are consultant forensic psychiatrists at St Andrew's, a charity providing specialist NHS mental healthcare in Northampton, where Suja Sreedharan is a senior specialty doctor. Correspondence Dr Paula Murphy, St Andrew's, Billing Road, Northampton NN1 5DG, UK. Email: paulamurphy@doctors.org.uk

\begin{abstract}
SUMMARY
High-dose antipsychotics are sometimes used in clinical practice when patients fail to respond to treatment at standard doses. Owing to the potential physical complications associated with this, strict adherence to physical health monitoring is essential. Challenges arise for clinicians when patients refuse to cooperate with this monitoring. We discuss different interventions to overcome this problem, many of which are coercive in nature, and consider their professional, ethical and legal implications. We include a flow diagram to assist clinicians in their documentation and decision-making in these circumstances as well as case vignettes showing when monitoring under restraint is and is not justifiable.

\section{LEARNING OBJECTIVES}

- Know what the physical complications of highdose antipsychotics are

- Be aware of the different ways of encouraging cooperation with physical health monitoring in patients who refuse it and of the legal frameworks under which they are governed

- Be knowledgeable on the issue of proportionality and its relevance to human rights

\section{DECLARATION OF INTEREST}

None
\end{abstract}

High-dose antipsychotics are prescribed for patients who fail to respond to standard doses and treatment (Taylor 2012), even though there is little evidence to show that higher doses are any more effective. High dose is defined as a total daily dose of a single antipsychotic that exceeds the upper limit stated in the British National Formulary (BNF; Joint Formulary Committee 2014) or a total daily dose of two or more antipsychotics (each dose expressed as a percentage of its BNF upper limit) that exceeds $100 \%$. This is regardless of whether antipsychotics are being used regularly or on an as-required (pro re nata or p.r.n.) basis. For the majority of patients, high-dose antipsychotics are prescribed through polypharmacy (Royal College of Psychiatrists 2014).

\section{Prevalence of high-dose prescribing}

In the largest survey of high-dose prescribing, around half of the patients were prescribed highdose antipsychotics as a result of p.r.n. use, even though many of these were not administered on the day of the survey (Harrington 2002).

Although it is recommended that high-dose antipsychotics should be reserved for exceptional clinical practice where standard treatments, including clozapine, have failed (Taylor 2012), around one-quarter to one-third of in-patients are prescribed high-dose antipsychotics, and the figure may be even higher in psychiatric intensive care units, rehabilitation wards and forensic units (Paton 2008; Royal College of Psychiatrists 2014). A 2011 survey of 651 patients revealed that high-dose antipsychotic use was associated with male gender, history of repeated admissions, use of depot medications, use of any antipsychotic combinations, use of at least one second-generation antipsychotic agent, provision of antimuscarinics, prescription of physical medications, and being admitted for over 36 days (Tungaraza 2011).

Despite the lack of firm evidence in favour of high-dose antipsychotics, there are a number of reasons why high-dose therapy is still used. First and foremost is the lack of response in treatmentresistant schizophrenia (Haw 2003; Paton 2007; Stahl 2012). Stahl argues that 'real world psychiatrists' have to treat illness where standard treatment has failed and reports that support for high-dose antipsychotic use is coming from 'practicebased evidence' (Stahl 2012). Other reasons include not wishing to change medication in a patient who is reasonably well and safety considerations such as increase in violence (Haw 2003).

\section{What is high-dose prescribing?}

Although the term 'high dose' is well defined - a total daily dose of a single antipsychotic or a total daily dose of two or more antipsychotics that exceeds the BNF maximums - it is worth remembering how BNF maximum doses are decided, i.e. from data derived from phase three 
clinical trials. This raises the important question of 'generality', as it is unlikely that factors such as gender, age, ethnic group and symptom severity are adequately controlled in such trials. How representative is a BNF maximum dose that has arisen from a clinical trial involving young male out-patients with moderate symptoms, if the patient before you is a middle-aged female inpatient with treatment-resistant symptoms? There are also a number of pharmacokinetic factors that affect plasma levels of medication. In some individuals, above-BNF doses may be required to reach the same plasma level as observed in others who are receiving within-BNF doses of the same medication. An important example is the effect of metabolism by cytochrome P450 enzymes, which may be affected by genetic differences and enzyme inhibition (e.g. CYP1A1 inhibition by St John's Wort) and induction (e.g. CYP1A2 induction by tobacco smoking).

\section{When to prescribe high-dose antipsychotics}

Before high-dose medication is considered for any patient, it is essential that all other evidencebased strategies, including clozapine, have been exhausted. If high-dose treatment gives no appreciable improvement within 3 months, a

BOX 1 Recommendations when prescribing high-dose antipsychotics

- Discuss the proposed strategy with the patient, family, carers and advocates

- Consult with the wider multidisciplinary team

- Document the decision in the patient's clinical notes, including the benefits of the strategy and when and how the outcome will be assessed

- Limit dose escalation to small increments, allowing adequate time for treatment response

- Monitor the total dose of regular and as-required (p.r.n.) medication and record it as a percentage of the BNF maximum

- Place alerts to notify the wider clinical team of the high-dose regime

- Consider potential drug interactions on a regular basis

- Before commencing high-dose therapy, carry out a baseline ECG to exclude cardiac contraindications such as long OT syndrome

- Repeat ECGs after a few days and every 1-3 months in the early stages of high-dose therapy

- Record target symptoms, response and side-effects, using validated scales where available

(Based on Royal College of Psychiatrists 2006; Taylor 2012) standard dose should be returned to (Royal College of Psychiatrists 2014). Of course, there are cases where patients refuse oral medication altogether, thereby restricting the available treatment options.

When the decision is taken to prescribe high-dose antipsychotics, it is important to remember that the majority of their side-effects are dose-related and that incidence and severity are greater with high-dose prescribing (Baldessarini 1988; Bollini 1994). Side-effects may be serious and they include QTc prolongation and sudden cardiac death as well as movement disorders, postural hypotension and anticholinergic symptoms (Taylor 2012). Some side-effects can be monitored clinically, but others require close monitoring with investigations such as blood tests and electrocardiograms (ECGs). Despite the significant risks of high-dose antipsychotics, physical monitoring in patients receiving them is poor (Harrington 2002).

The decision to prescribe high-dose antipsychotics should involve an individualised riskbenefit assessment and the recommendations outlined in Box 1 should be followed.

\section{Physical health monitoring when patients refuse to cooperate}

The importance of monitoring physical health cannot be overstated (Royal College of Psychiatrists 2014). However, in practice, monitoring is challenging when a patient refuses to cooperate (Tyson 1999). There may be a number of reasons why a patient will not cooperate with monitoring, but lack of insight is likely to be the most significant. This is not surprising, given that high-dose antipsychotic medication is used to treat severe illness and that severe illness is associated with reduced insight (Sevy 2004). Often, there is no option to discontinue high-dose antipsychotic treatment because the risk of relapse is too great. Concerns about partial treatment are equally valid, given the association between untreated psychosis and clinical outcome (Marshall 2005). Last, where untreated illness is associated with an increased risk of self-harm and violence, the justification for dose reduction is even weaker.

If the patient's risk-benefit assessment favours the continuation of high-dose antipsychotics, every effort must be made to ensure appropriate physical health monitoring. However, should the patient refuse to cooperate, what options are available to the treating team and where do the boundaries lie in terms of the professional, ethical and legal implications? There are no published guidelines to indicate what procedures should be followed when a patient refuses to comply. As unsavoury as it may sound, coercive practice is often employed in such 
instances. Coercion in psychiatry has been defined as 'any action or threat of actions which compels the patient to behave in a manner inconsistent with his own wishes' (Whittington et al 2006, cited in Georgieva 2012).

Coercion remains a controversial subject and there has been much debate about its use and form. Data from the Care Quality Commission suggest that coercive practices are being used unnecessarily in some cases. For example, more than $30 \%$ of patients on community treatment orders did not have a history of refusing to cooperate with their treatment, including medication. It was suggested that mental health professionals were using 'defensive practices' because of perceived risks (Care Quality Commission 2010: p. 44).

\section{The four Cs}

In the absence of guidance on how to encourage cooperation with physical health monitoring in patients who are receiving high-dose antipsychotics, we will consider a number of approaches, some coercive by nature (which raises important ethical and legal challenges). We think of these as the four Cs (Fig. 1):

- continual encouragement

- compliance therapy

- contingency management

- control and restraint.

We have also developed a flow diagram to guide clinicians when working with uncooperative patients (Fig. 2). The purpose of this is to help prevent overly coercive practices that might infringe the patient's human rights.

\section{Continual encouragement}

Non-concordance with medical advice is not unique to psychiatry (Osterberg 2005), but encouraging adherence is even more challenging in psychiatry because many patients have limited insight into

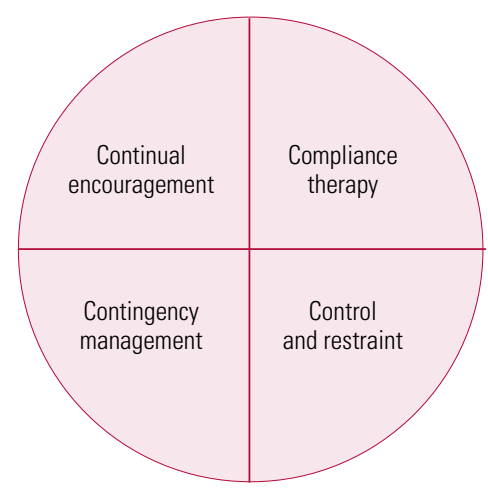

The four Cs of compliance management. their illnesses and their need for treatment. It is for this reason that communication and therapeutic alliance play such a significant part. Perhaps an approach all too easily overlooked when trying to improve adherence is continual encouragement. It is vital for the whole multidisciplinary team to engage in this: it should not be the sole responsibility of the prescriber. For example, conversations with the patient about their physical health can be casually incorporated into activities with the patient during sessions with occupational or nursing staff. Particular members of staff may be better placed to do this and should be employed accordingly. Likewise, the patient's relatives, carers or friends might be in a good position to help in encouraging cooperation with physical health investigations and should be involved in care plans wherever possible. Although continual encouragement may be seen as common sense, for patients who take high-dose antipsychotic medication and who refuse physical health monitoring, this simple but important method is sometimes easily forgotten or overlooked.

\section{Compliance therapy}

More formal approaches include the use of compliance (adherence) therapy. This combines cognitive-behavioural techniques, motivational interviewing and psychoeducation to target treatment adherence. An early study of the effectiveness of the approach in a sample of inpatients with psychotic disorders allocated 39 individuals to compliance therapy and 35 to nonspecific counselling (Kemp 1998). Significant advantages were found in the compliance therapy group on measures of insight, attitudes to treatment and observer-related adherence. However, when this study was replicated (O'Donnell 2003; Gray 2006), there was no difference in adherence rates between the sample and control groups. More recently, a large review of interventions to improve adherence to antipsychotic medication for schizophrenia showed that interventions that are longer in duration, offer more sessions and provide continuous focus on adherence are more successful in improving adherence than interventions of short duration (Barkhof 2012). Although compliance therapy showed early promise in improving adherence to treatment in schizophrenia, little is known about its effect on patients' adherence to high-dose antipsychotic monitoring.

\section{Contingency management}

Contingency management involves the reward or, less commonly, the punishment of particular behaviours. It is more often associated with 


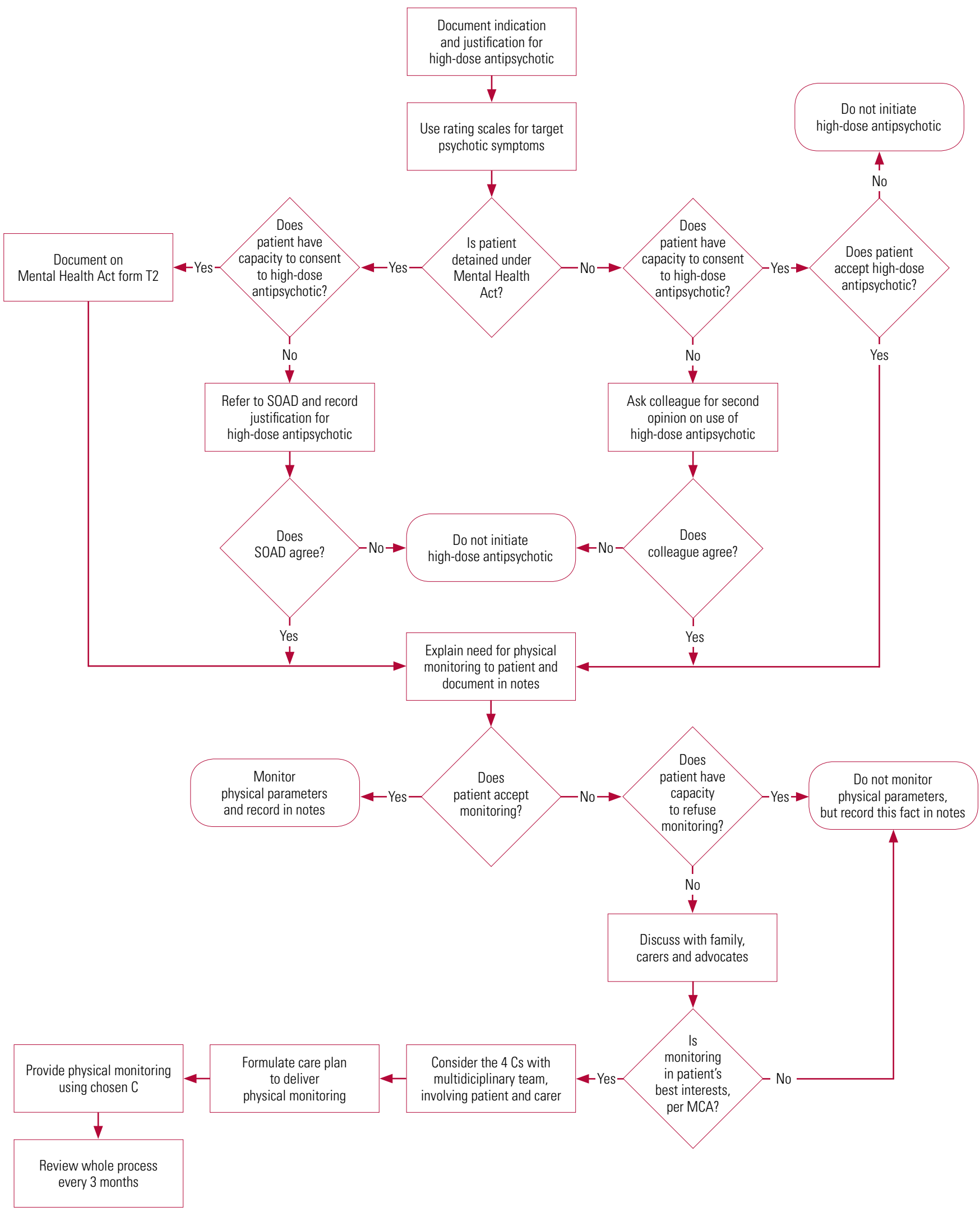

Reaching the decision to prescribe high-dose antipsychotics and subsequent physical health monitoring. MCA, Mental Capacity Act 2005; SOAD, second opinion appointed doctor; the $4 \mathrm{Cs}$ : continual encouragement, compliance therapy, contingency management, and control and restraint. 
addiction, where it has been used successfully to reduce drug use (Petry 2012). Its use has also been extended to the treatment of medical conditions such as pressure ulcers (Adkins 1999).

Where incentives are used, it is the target behaviour, for example adhering to monitoring, that is rewarded. To be effective, rewards should be tangible. They usually include vouchers, gift cards or cash. Where punishments are used, it is the absence of the target behaviour, for example refusing to adhere to monitoring, that is punished. Examples of punishment include financial losses and loss of privileges. In a study of the use of contingency management in smoking cessation, Romanowich et al (2013) showed that losses motivate behaviour more than equal gains. Participants who stood to lose vouchers for each day of failed abstinence were more likely to achieve a day of abstinence than participants who stood to gain a voucher for each day of successful abstinence.

Informed by the international evidence base, the National Institute for Health and Care Excellence (NICE) recommended the use of contingency management by drug services in the UK: 'to reduce illicit drug use and/or promote engagement with services for people receiving methadone maintenance treatment' (NICE 2007: para. 1.4.1.1).

\section{Concerns about contingency management}

Contingency management remains a controversial approach in the UK and despite the recommendations made by NICE, its uptake in drug services has been limited (Petry 2006; National Treatment Agency for Substance Misuse 2007; NICE 2007; Sinclair 2011). Reasons include differences in opinions of service providers on, for example, how contingency management aligns to the philosophy of substance misuse services, wider ethical concerns and the practicalities of implementation (Sinclair 2011) and its cost (Petry 2006).

Many ethical issues remain regarding its use for addictions, particularly concerns that money-based incentives might be used by patients to buy more drugs and that the concept may mimic gambling (Petry 2006). In addition, the view of 'Why pay people to do what they should be doing anyway?' resonates with many. According to Petry (2006), such objections may be 'somewhat tempered by pragmatic and Machiavellian principles of doing whatever works best for this difficult-to-treat and often disenfranchised population'. By the same rationale, similar principles could apply to the hard-to-reach psychiatric population who refuse to allow physical health monitoring. Indeed, contingency management has been applied in areas other than retaining substance misuse patients in treatment. It has been used to reinforce adherence to medication in patients with AIDS and sexually transmitted diseases (Rosen 2007), in helping individuals to lose weight (Volpp 2008), in increasing adherence to exercise regimens by people with substance use disorders (Weinstock 2008) and in decreasing smoking in people with schizophrenia (Roll 1998).

Although contingency management raises important ethical considerations, it is worthwhile exploring this approach further, especially when the benefits of cooperating with physical health monitoring by patients who lack capacity outweigh the ethical costs of using money-based incentives.

\section{Control and restraint}

Five types of restraint are recognised in healthcare (Gallagher 2011) (Box 2):

- physical

- mechanical

- technological

- chemical

- psychological.

In theory, more than one type might be necessary to improve cooperation with physical health monitoring. Examples include making a patient's leave contingent on their ongoing adherence to monitoring (this is an example of psychological restraint); sedating a patient to facilitate an electrocardiogram (ECG); physically restraining a patient to obtain bloods; secluding a patient for a period of time after obtaining bloods to reduce the risk of violence; and using rapid tranquillisation as chemical restraint. In practice, however, some

B0X 2 Types of restraint

Physical restraint

Holding the patient by one or more persons

Mechanical restraint

The use of equipment such as straps and belts

Technological restraint

Uses tagging or door alarms

Chemical restraint

The use of medication to sedate the patient

Psychological restraint

Telling the patient that they are not allowed to do something or depriving them of choices 
types of restraint are rarely used. For example, it is rare that an ECG will be done under sedation.

The decision to use restraint to enable an intervention (such as a blood test) requires a carefully documented assessment weighing up the risks and benefits of intervening under restraint versus not intervening at all, taking into consideration the clinical, professional, ethical and legal implications of each. Such considerations safeguard against any deprivation of the patient's liberty and the permissibility of the intervention.

\section{Legislation governing restraint}

To ensure that unnecessary restraint does not take place, it is essential that healthcare practitioners understand the legal framework governing its use. In England and Wales, the use of restraint in mental health settings is governed by the Mental Health Act 1983, as amended in 2007, and the Mental Capacity Act 2005.

\section{The Mental Health Act}

If a person is detained under the Mental Health Act, staff may use physical restraint to exercise a degree of control over them at times. Although physical restraint is not defined under the Act itself, guidance on its use is provided in Chapter 15 of the associated Code of Practice (Department of Health 2008). According to the Code, common reasons for needing to consider physical restraint are: physical assault; dangerous, threatening or destructive behaviour; self-harm or risk of physical injury by accident; extreme and prolonged overactivity that is likely to lead to physical exhaustion; and attempts to abscond (where the patient is detained under the Act). The Code specifies that the method of restraint used 'must balance the risk to others with the risk to the patient's own health and safety and must be a reasonable, proportionate and justifiable response to the risk posed by the patient' (Department of Health 2008: para. 15.19). Mental health legislation allows compulsory treatment (which may include physical health monitoring) only for a mental disorder and conditions ancillary to the mental disorder. Examples of physical complications of mental disorder that can be legally treated include self-harm due to personality disorder or overdose due to depression. It does not provide authority to proceed where the treatment is for a condition unrelated to the mental disorder.

\section{The Mental Capacity Act}

In situations where the physical health of the patient is at risk, it is necessary to determine the capacity of the individual with respect to their ability to make a decision on their treatment. In accordance with the Mental Capacity Act, to demonstrate capacity, individuals should be able to:

- understand (with the use of communication aids, if appropriate) in simple language what the medical treatment is, its purpose and nature and why it is being proposed

- understand its principal benefits, risks and alternatives

- understand in broad terms the consequences of not receiving the proposed treatment

- retain the information for long enough to use it and weigh it in the balance to arrive at a decision

- communicate the decision (by any means).

Should the patient lack capacity, restraint may be used if it is felt to be in the patient's best interests. Referring to section 6(4) of the Mental Capacity Act, the Code of Practice for the Act describes restraint as 'The use or threat of force to help do an act which the person resists, or the restriction of the person's liberty of movement, whether or not they resist' (Department for Constitutional Affairs 2007: p. 290).

\section{Restraint and proportionality}

Restraint can be used under the Mental Capacity Act only if two conditions are satisfied:

1 the person wishing to restrain the patient reasonably believes that restraint is necessary to prevent harm

2 the restraint is a proportionate response to the likelihood of the patient suffering harm and the seriousness of that harm.

Therefore, in considering the physical health monitoring of patients on high-dose antipsychotics, the harm would be the adverse physical effects of the medication. The argument for using restraint would be dependent on whether the clinician believed it to be necessary to avoid that harm. The first condition is perhaps easier to satisfy than the second, which is made more difficult by the notion of proportionality. On one hand, the seriousness of the potential adverse events and interactions are such that restraint is proportionate. On the other hand, there may be a number of specific factors that would lead a clinician to hold a different view; these might include the age, gender, size and medical history of the patient in question. A proportionate response under the Mental Capacity Act can be defined as 'using the least intrusive type and the minimum amount of restraint to achieve the objective, in the best interests of the patient lacking capacity' (British Medical Association 2008: p. 19). 
The issue of proportionality therefore arises under both the Mental Capacity Act and the Mental Health Act, as demonstrated in the Code of Practice for each. The issue hinges on human rights principles. All UK legislation is expected to be compatible with the Human Rights Act 1998, as required by the European Convention on Human Rights (Curtice 2011).

To be compatible with the Human Rights Act, proportionality must be considered, as the Act requires 'a search for a fair balance between the demands of the general interest of the community and the requirements of the protection of the individual's fundamental rights' that is inherent in the whole Convention (Soering v. UK (1989) 11 EHRR 439, cited in Curtice 2011). This is central to the case law that has produced a fourpronged test of proportionality, the 'Huang test' (Curtice 2011):

1 Does the policy (or measure) in question pursue a sufficiently important objective?

2 Is the rule or decision under review rationally connected with that objective?

3 Are the means adopted no more than necessary to achieve that objective?

4 Does the measure achieve a fair balance between the interests of the individual(s) affected and the wider community? (i.e. does the measure constitute a proportionate means of achieving a legitimate aim?)

Clearly, restrictive measures should always be a last resort and alternatives to physical restraint should be tried first. The use of physical restraint must be justified by demonstrating that it is necessary and that the patient is likely to suffer harm unless proportionate restraint is used.

\section{Guidelines on using physical restraint}

The decision to use physical restraint should not be taken lightly, not least because of the potential problems associated with this type of intervention. In 1998, inquiries into the death of David Bennett, a patient in a medium secure facility, found that he died as a consequence of prolonged face-down physical restraint and the amount of force used by members of staff during the incident, which caused positional asphyxia. The results of the inquiry were published and recommendations included that 'under no circumstances should any patient be restrained in a prone position for a longer period than three minutes' and 'a national system of training in restraint and control should be established as soon as possible and, at any rate, within twelve months of the publication of this report' (Norfolk, Suffolk and Cambridgeshire
BOX 3 Guidelines on the use of physical restraint

Physical restraint should:

- be used only for as long as is absolutely necessary

- use a recognised technique that does not depend on the deliberate application of pain

- be carried out by people who have received appropriate training in the use of restraint techniques

Throughout the period when physical restraint is being used:

- a doctor should be quickly available to attend an alert by staff members

- staff should continue to employ de-escalation

- staff should be alert to the risk of respiratory or cardiac distress

- emergency resuscitation devices should be readily available in the area where the restraint is taking place

- the patient's physical and psychological well-being should be monitored

(Department of Health 2008)

Strategic Health Authority 2003: p. 67). For such reasons additional guidance on the use of physical restraint has been produced as set out in Box 3. Despite such guidance, there have been 13 restraint-related deaths of patients detained under the Mental Health Act since the death of David Bennett in 1998 (MIND 2013).

\section{The dangers of restraint}

In addition to the very real danger of death, physical restraint can cause patients physical injuries and psychological problems. A recent inquiry by MIND documented that in 2012 alone there were almost 1000 incidents of physical injury following physical restraint in hospital settings in England (MIND 2013). In addition to the physical injuries, many patients reported physical restraint as being humiliating, distressing and disempowering and some commented on the long-term psychological impact from an episode of physical restraint.

It is likely that physical restraint also causes a loss of trust between doctor and patient and therefore its longer-lasting effects need to be included when weighing up the risks and benefits.

\section{Ethical questions}

To explore the ethical issues around restraint, we will use Beauchamp \& Childress's four principles of biomedical ethics: autonomy, beneficence, non-maleficence and justice (Beauchamp 2001). Although restraint contradicts the principle of autonomy - that the patient has the right to refuse 
or choose their treatment - the principle relies on the premise that individuals are competent (Donnelly 2012). Notwithstanding the risk of medical paternalism (Thomasma 1983), for a patient who lacks capacity, the right to autonomy may be outweighed by the principle of beneficence, which states that a practitioner should act in the patient's best interests. Although beneficence may justifiably override the principle of autonomy in this scenario, the principle of non-maleficence is more complicated. For the refusing patient, restraint may be perceived as harm and would contradict non-maleficence, the requirement to 'do no harm'. However, the failure to restrain a patient to safely monitor high-dose antipsychotic therapy may cause further harm. The principle of justice requires the healthcare professional to act fairly and be able to justify actions taken, thereby emphasising the importance of meticulous notekeeping with a clear rationale for any actions.

Of course, the legal implications of not monitoring the physical effects of high-dose antipsychotic prescribing and allowing the patient to come to harm should also be considered in the riskbenefit assessment, as it could constitute clinical negligence in the eyes of the law. For a claim of clinical negligence to be upheld, the following conditions must hold (MIND Legal Unit 2013):

1 a duty of care exists

2 the duty of care has been breached

3 the breach of duty has resulted in harm

4 the harm has resulted in damage or other losses.

Medical professionals have a duty of care to all their patients and physical health monitoring when prescribing high-dose antipsychotics is necessary because of the well-documented health risks associated with the practice. Therefore, if a patient comes to harm through physical health complications secondary to high-dose antipsychotic use, even if they refused to allow monitoring, it could be viewed as a neglect of the duty of care to the patient. This will of course depend on clear documentation of treatment rationale and the reasons why the monitoring was not possible.

\section{Case vignettes}

The following fictitious case vignettes provide examples of when restrictive practices may or may not be justified.

\section{Case vignette 1: Justifiable restraint}

$\mathrm{Mr}$ X, a 45-year-old man with treatment-resistant paranoid schizophrenia, is detained in a medium secure unit. He has responded poorly to a number of antipsychotics in the past. He has refused clozapine on several occasions because he does not believe that he needs it. His best treatment response has been achieved with his current regime of zuclopenthixol decanoate $300 \mathrm{mg}$ weekly by depot injection and oral aripiprazole $20 \mathrm{mg}$ daily. Owing to extrapyramidal side-effects, he has been unable to tolerate higher doses of the depot. He refuses physical health monitoring, stating that there is nothing wrong with him. Following months of continual encouragement from the multidisciplinary team, he finally allowed routine blood testing, which found a random glucose level of $39 \mathrm{mmol} / \mathrm{L}$. He refused any further investigation, stating that there was nothing wrong with his blood glucose.

In this case, the first step is to assess the patient's capacity and record this fully in the notes. This patient lacks capacity, as he does not believe that the high glucose reading is a problem. The clinical team must therefore act in the patient's best interests. Given the potential seriousness of the situation, it is important that investigations take place urgently. As he does not have capacity, restraint and treatment under the Mental Capacity Act may be used if necessary. This would be justifiable because not treating him could lead to diabetic coma. Restraint to do further blood tests could therefore be considered a proportionate response to the situation. To do nothing, and allow the patient to come to harm, would be clinically negligent.

\section{Case vignette 2: Restraint cannot be justified}

Mr $\mathrm{Y}$ is a 26-year-old man with a history of treatment-resistant schizophrenia. $\mathrm{He}$ is being treated with olanzapine $20 \mathrm{mg}$ daily and risperidone $8 \mathrm{mg}$ daily. He was receiving clozapine but it was stopped when he developed myocarditis. The myocarditis subsequently resolved. He has started to express beliefs that the ECG machine is able to record his thoughts and he has been refusing to comply with ECG monitoring. He does not accept that monitoring of his heart is important or necessary. There are concerns because he is on high-dose antipsychotics and has a history of cardiac problems. His vital signs have been normal and he has not presented with any obvious health problems on the ward.

In this case, again the first step is to assess the patient's capacity. He has expressed delusional reasons for refusing an ECG and so does not have capacity to make the decision to refuse monitoring. Treatment should therefore be carried out in the patient's best interests. The main concern is that he is on high-dose antipsychotics and there is a risk of prolongation of the QTc interval, which is compounded by his history of cardiac problems. The fact that he does not have any signs or symptoms is reassuring. However, the rationale for taking an ECG reading under restraint is questionable. The intervention would be difficult 
MCO answers

$1 \mathrm{c} \quad 2 \mathrm{e} \quad 3 \mathrm{~d} \quad 4 \mathrm{e} \quad 5 \mathrm{~b}$ to perform and the reading would be compromised by artefact. Furthermore, the risks associated with restraining this patient might outweigh any benefits, given his history of cardiac disease. Any ECG, therefore, would require sedation or general anaesthetic, both of which pose different risks and thus may be deemed to be a disproportionate response to the situation. Although the ECG is important, it does not need to be done urgently in this case and other methods could be employed to encourage cooperation, such as a combination of continual encouragement and compliance therapy.

\section{Conclusions}

High-dose antipsychotics should be prescribed only when all other options, including clozapine, have been exhausted. The use of high-dose antipsychotics requires strict adherence to physical health monitoring because of the serious sideeffects of using doses above BNF maximums. Patients who refuse to comply with this monitoring are at risk of potentially fatal outcomes. Sometimes a patient's refusal is embedded in delusional beliefs, for example believing that their blood will be used for experiments or that the sphygmomanometer causes muscle weakness. Capacity therefore plays an important role in determining what the next steps should be. Coercive practice, although deemed unpopular by many, can be considered as a necessary evil when meeting the best interests of patients who lack capacity. Coercive techniques vary in degree and range from continual encouragement to control and restraint.

It is essential that clinicians work within the legal frameworks that underpin these practices, namely the Mental Health Act 1983 and Mental Capacity Act 2005, both of which must comply with the Human Rights Act 1998. The question of proportionality is considered pivotal to human rights and it is essential that the least restrictive method is always used. The proportionality (Huang) test has been suggested as a useful tool in clinical practice (Curtice 2011). It is essential that multidisciplinary teams work together to ensure that they have demonstrably considered all the alternative interventions before moving on to more coercive methods. A structured approach, as outlined in Fig. 2, can be used to help clinicians in their decision-making and ensure that they have considered all the relevant facets. In particularly complex cases, it may be prudent to seek professional or legal advice and certainly this is to be encouraged, given the potential infringement of a patient's human rights should overly coercive practices be used.

\section{References}

Adkins VK, Mathewson C, Ayllon T, et al (1999) The ethics of using contingency management to reduce pressure ulcers: data from an exploratory study. Ostomy/Wound Management, 45(3): 56-8.

Baldessarini RJ, Cohen BM, Teicher MH (1988) Significance of neuroleptic dose and plasma level in the pharmacological treatment of psychoses. Archives of General Psychiatry, 45: 79-91.

Barkhof E, Meijer CJ, de Sonneville LM, et al (2012) Interventions to improve adherence to antipsychotic medication in patients with schizophrenia: a review of the past decade. European Psychiatry, 27: 9-18.

Beauchamp TL, Childress JF (2001) Principles of Biomedical Ethics. Oxford University Press.

Bollini P, Pampallona S, Orza MJ, et al (1994) Antipsychotic drugs: is more worse? A meta-analysis of the published randomized control trials. Psychological Medicine, 24: 307-16.

British Medical Association (2008) Card 7: Restraint. In Mental Capacity Act Toolkit. BMA.

Care Quality Commission (2010) Monitoring the Use of the Mental Health Act in 2009/10. COC.

Curtice M, Bashir F, Khurmi S, et al (2011) The proportionality principle and what it means in practice. Psychiatrist, 35: 111-6.

Department for Constitutional Affairs (2007) Mental Capacity Act 2005 Code of Practice. TSO (The Stationery Office).

Department of Health (2008) Code of Practice: Mental Health Act 1983. TSO (The Stationery Office).

Donnelly M (2012) From autonomy to dignity: treatment for mental disorders and the focus for patient rights. Law in Context, 26: 37-61.

Gallagher A (2011) Ethical issues in patient restraint. Nursing Times, 107(9): 18-20.

Georgieva I (2012) Coercive Interventions during Inpatient Psychiatric Care: Patient's Preference, Prevention and Effects. Erasmus University Rotterdam.

Gray R, Leese M, Bindman J, et al (2006) Adherence therapy for people with schizophrenia: European multicentre randomised controlled trial. British Journal of Psychiatry, 189: 508-14.

Harrington M, Lelliott P, Paton C, et al (2002) The results of a multi-centre audit of the prescribing of antipsychotic drugs for in-patients in the UK. Psychiatric Bulletin, 26: 414-8.

Haw C, Stubbs J (2003) Combined antipsychotics for 'difficult-to-manage' and forensic patients with schizophrenia: reasons for prescribing and perceived benefits. Psychiatric Bulletin, 27: 449-52.

Joint Formulary Committee (2014) British National Formulary (68). BMJ Group \& Pharmaceutical Press.

Kemp R, Kirov G, Everitt B, et al (1998) Randomised controlled trial of compliance therapy: 18-month follow-up. British Journal of Psychiatry, 172: 413-9.

Marshall M, Lewis S, Lockwood A, et al (2005) Association between duration of untreated psychosis and outcome in cohorts of first-episode patients: a systematic review. Archives of General Psychiatry, 62: 975-83.

MIND (2013) Mental Health Crisis Care: Physical Restraint in Crisis. A Report on Physical Restraint in Hospital Settings in England. MIND.

MIND Legal Unit (2013) Clinical Negligence. MIND (http://www.mind. org.uk/information-support/legal-rights/clinical-negligence). Accessed 16 Oct 2014

National Institute for Health and Clinical Excellence (2007) Drug Misuse: Psychosocial Interventions (NICE Clinical Guideline CG51). NICE.

National Treatment Agency for Substance Misuse (2007) Are Contingency Management Principles Being Implemented in Drug Treatment in England? NTA. 
Norfolk, Suffolk and Cambridgeshire Strategic Health Authority (2003) Independent Inquiry into the Death of David Bennett. Norfolk, Suffolk and Cambridgeshire SHA

O'Donnell C, Donohoe G, Sharkey L, et al (2003) Compliance therapy: a randomised controlled trial in schizophrenia. BMJ, 327: 834.

Osterberg L, Blaschke T (2005) Adherence to medication. New England Journal of Medicine, 353: 487-97.

Paton C, Whittington C, Barnes TR, et al (2007) Augmentation with a second antipsychotic in patients with schizophrenia who partially respond to clozapine: a meta-analysis. Journal of Clinical Psychopharmacology, 27: 198-204

Paton C, Barnes TRE, Cavanagh M-R, et al (2008) High-dose and combination antipsychotic prescribing in acute adult wards in the UK: the challenges posed by p.r.n. prescribing. British Journal of Psychiatry, 192: 435-9.

Petry NM (2006) Contingency management treatments. British Journal of Psychiatry, 189: 97-8.

Petry NM, Barry D, Alessi SM, et al (2012) A randomized trial adapting contingency management targets based on initial abstinence status of cocaine-dependent patients. Journal of Consulting and Clinical Psychology, 80: 276-85.

Roll JM, Higgins ST, Steingard S, et al (1998) Use of monetary reinforcement to reduce the cigarette smoking of persons with schizophrenia: a feasibility study. Experimental and Clinical Psychopharmacology, 6: 157-61

Romanowich P, Lamb RJ (2013) The effect of framing incentives as either losses or gains with contingency management for smoking cessation. Addictive Behaviors, 38: 2084-8.

Rosen MI, Dieckhaus K, McMahon TJ, et al (2007) Improved adherence with contingency management. AIDS Patient Care and STDs, 21: 30-40.
Royal College of Psychiatrists (2006) Consensus Statement on HighDose Antipsychotic Medication (Council Report CR138). Royal College of Psychiatrists.

Royal College of Psychiatrists (2014) Consensus Statement on HighDose Antipsychotic Medication (College Report CR190). Royal College of Psychiatrists.

Sevy S, Nathanson K, Visweswaraiah H, et al (2004) The relationship between insight and symptoms in schizophrenia. Comprehensive Psychiatry, 45: 16-9.

Sinclair JM, Burton A, Ashcroft R, et al (2011) Clinician and service user perceptions of implementing contingency management: a focus group study. Drug and Alcohol Dependence, 119: 56-63.

Stahl SM (2012) Antipsychotic polypharmacy: never say never, but never say always. Acta Psychiatrica Scandinavica, 125: 349-51.

Taylor D, Paton C, Kapur S (2012) The Maudsley Prescribing Guidelines in Psychiatry (11th edn). Wiley-Blackwell.

Thomasma DC (1983) Beyond medical paternalism and patient autonomy: a model of physician conscience for the physician-patient relationship. Annals of Internal Medicine, 98: 243-8.

Tungaraza TE, Zahid U, Venkataramaiah B (2011) Polypharmacy and high-dose antipsychotics at the time of discharge from acute psychiatric wards. Psychiatrist, 35: 288-92.

Tyson PJ, Mortimer AM, Wheeler JA (1999) High-dose antipsychotic treatment in clinical practice: a review, audit and survey of consultant psychiatrist opinions. Psychiatric Bulletin, 23: 661-4.

Volpp KG, John LK, Troxel AB, et al (2008) Financial incentive-based approaches for weight loss: a randomized trial. JAMA, 300: 2631-7.

Weinstock J, Barry D, Petry NM (2008) Exercise-related activities are associated with positive outcome in contingency management treatment for substance use disorders. Addictive Behaviors, 33: 1072-5.

\section{MCQs}

Select the single best option for each question stem

1 Which of the following is a complication of prescribing high-dose antipsychotics?

a prolonged PR interval

b peaked t-wave

c prolonged QTc interval

d inverted ST segment

e shortened QTc interval.

\section{Which of the following is not a recognised} type of restraint?

a physical

b technological

c chemical

d psychological

e social.

\section{Restraint under the Mental Health Act} must be:

a timely, justifiable and proportionate b timely, reasonable and proportionate

c timely, proportionate and necessary

d reasonable, justifiable and proportionate

e necessary, measurable and justifiable.

4 Which of the following is not a typical method for gaining adherence to treatment?

a compliance therapy

b contingency management

c control and restraint

d continual encouragement

e cognitive analytic therapy.

5 In which of the following scenarios would you intervene to monitor high-dose antipsychotic use?

a patient detained, patient lacks capacity to consent to treatment, high-dose antipsychotic authorised by second opinion appointed doctor (SOAD), patient accepts monitoring b patient detained, patient lacks capacity to consent to treatment, high-dose antipsychotic authorised by $S O A D$, patient refuses monitoring, patient lacks capacity to make such decision, monitoring is in patient's best interests

c patient detained, patient lacks capacity to consent to treatment, high-dose antipsychotic authorised by SOAD, patient refuses monitoring and has capacity to refuse

$\mathrm{d}$ patient not detained, patient consents to treatment with high-dose antipsychotics, patient accepts monitoring

e patient not detained, patient lacks capacity to consent to treatment, colleague agrees to high-dose antipsychotic use, patient accepts monitoring. 